\title{
HAND GRIP PRESSURE IN OLDER PEOPLE
}

\author{
BY \\ W. F. ANDERSON AND N. R. COWAN \\ Consultative Health Centre for Older People, Rutherglen, Lanarkshire
}

It is postulated that grip strength is of value in estimating progress after illness, that it can aid in the seeking out of early pathological processes involving the related musculature, and that it is of use in the assessment of certain generalized diseases, for example, the myopathy associated with neoplasm and thyrotoxicosis. However, there is a clear lack of information concerning "normal" grip strength in older persons and its use in geriatric practice. Grip strength has been considered in the past mainly in terms of age and zero order correlation, with inadequacy of numbers in older ages (Ruger and Stoessiger, 1927; Elderton and Moul, 1928; Fisher and Birren, 1947). Burke, Tuttle, Thompson, Janney, and Weber (1953), with only 27 subjects in the age range 60-79 years, state that the average grip strength of males at 79 years is $32.2 \mathrm{~kg}$. and that this is about the same as that at age 12 to 15 years. This paper has therefore a two-fold purpose: to provide standards of hand grip pressure for healthy older people at given weight and age, and thereby to encourage the routine measurement of grip strength in geriatric practice.

\section{METHODS}

The data relate to 362 men and 394 women seen at the Rutherglen Consultative Health Centre for Older People (Anderson and Cowan, 1955). All were considered to be in good health after a detailed clinical examination, which included ophthalmoscopic examination and where necessary electrocardiographic assessment. In particular, hand injuries, left-handedness, a haemoglobin level under $11 \mathrm{~g}$. Sahli, or an apical systolic murmur louder than Grade 2 as described by Levine and Harvey (1949) excluded people from this series.

The hand grip pressure was measured with a "Meredith" dynamometer. Each individual was permitted three tries with each hand and the highest reading is the one recorded.

Body weight was measured with men and women wearing a minimum of clothing and no footwear. The weighing machine was of the Steelyard platform type reading accurately to $85 \mathrm{~g}$.

\section{REsults}

Table I shows that men have more powerful hand grips than women. The average hand grip pressures of men and women differ significantly for both hands (Right hand: mean difference $=9.2 \mathrm{~kg}$.; $\mathrm{s}=0.333912$; $\mathbf{t}^{\prime}=27.4 ; 5$ per cent. level $=1.96$. Left hand: mean difference $=9.1 \mathrm{~kg}$.; $\mathrm{s}=0.329895 ; \mathrm{t}^{\prime}=27.5 ; 5$ per cent. level =1.96). The test of significance used for these mean differences required to take account of the significant differences between the standard

TABLE I

MEANS, STANDARD DEVIATIONS, AND COEFFICIENTS OF VARIATION OF THE SEVERAL VARIABLES, BY SEX

\begin{tabular}{|c|c|c|c|c|c|c|c|c|c|c|}
\hline & & ble & & & & & Sex & Mean \pm S.E. & $\begin{array}{l}\text { Standard } \\
\text { Deviation }\end{array}$ & $\begin{array}{l}\text { Coefficient } \\
\text { of Variation }\end{array}$ \\
\hline \multirow{2}{*}{ Hand Grip Pressure (kg.) } & \multirow{2}{*}{$\cdots$} & \multirow{2}{*}{$\cdots$} & \multirow{2}{*}{$\cdots$} & \multirow{2}{*}{$\cdots$} & \multicolumn{2}{|c|}{ Right .. } & $\begin{array}{c}\text { Male } \\
\text { Female }\end{array}$ & $\begin{array}{l}39 \cdot 5 \pm 0 \cdot 28 \\
30 \cdot 3 \pm 0 \cdot 18\end{array}$ & $\begin{array}{l}5 \cdot 4 \\
3 \cdot 6\end{array}$ & $\begin{array}{l}13 \cdot 7 \\
11 \cdot 9\end{array}$ \\
\hline & & & & & Left & $\cdots$ & $\begin{array}{c}\text { Male } \\
\text { Female }\end{array}$ & $\begin{array}{l}37 \cdot 5 \pm 0 \cdot 28 \\
28 \cdot 4 \pm 0 \cdot 18\end{array}$ & $\begin{array}{l}5 \cdot 3 \\
3 \cdot 6\end{array}$ & $\begin{array}{r}14 \cdot 1 \\
-\quad 12 \cdot 6\end{array}$ \\
\hline Body weight (kg.) .. & $\cdots$ & $\cdots$ & $\cdots$ & $\cdots$ & $\cdots$ & $\cdots$ & $\begin{array}{c}\text { Male } \\
\text { Female }\end{array}$ & $\begin{array}{l}63 \cdot 0 \pm 0.49 \\
61 \cdot 6 \pm 0.62\end{array}$ & $\begin{array}{r}9 \cdot 4 \\
12 \cdot 4\end{array}$ & $\begin{array}{l}14 \cdot 9 \\
20 \cdot 1\end{array}$ \\
\hline Age (completed years) & $\cdots$ & $\cdots$ & $\cdots$ & $\cdots$ & $\cdots$ & $\cdots$ & $\begin{array}{c}\text { Male } \\
\text { Female }\end{array}$ & $\begin{array}{l}72 \cdot 4 \pm 0 \cdot 32 \\
70 \cdot 1 \pm 0 \cdot 35\end{array}$ & $\begin{array}{l}6 \cdot 9 \\
6 \cdot 9\end{array}$ & $\begin{array}{l}9 \cdot 5 \\
9 \cdot 8\end{array}$ \\
\hline
\end{tabular}


deviations of hand grip pressures by sex. The standard deviations are greater in men than women (Right hand: $\mathrm{F}=2.15 ; \mathrm{P}<0.001$. Left hand: $\mathrm{F}=2.05$; $\mathbf{P}<0.001)$.

The correlation Tables II and III show the relationship between the right hand grip pressure and $\log$ weight for men and women respectively. The corresponding correlation data for the left hand grip pressure follow a similar trend*. The distributions of hand grip pressures are not significantly skew (Right hand: men $\mathrm{t}=1.26$; women $\mathrm{t}=1.35$. Left hand: men $\mathrm{t}=1.44$; women $\mathrm{t}=0.56$ ) or kurtotic (Right hand: men $\mathrm{t}=-0.68$; women $\mathrm{t}=-0.37$.
Left hand: men $\mathrm{t}=-0.48$; women $\mathrm{t}=-0.21$ ). The distributions of body weight depart significantly from "normality". They are significantly skew (men $\mathrm{t}=2.74$; women $\mathrm{t}=3.24$ ) but are not significantly kurtotic (men $\mathrm{t}=-0.65 ;$ women $\mathrm{t}=0.81$ ). However, a logarithmic transformation of body weight data shows no significant departure from "normality" with regard to skewness (men $\mathrm{t}=0.08$; women $\mathrm{t}=-0.97$ ) or kurtosis (men $\mathrm{t}=-1.42$; women $\mathrm{t}=-0.50)$. The 0.01 level of significance is $t=2 \cdot 5758$. Consequently, a logarithmic transformation of body weight data is used in this analysis.

* The further correlation tables may be obtained from the authors.

TABLE II

DISTRIBUTION OF RIGHT HAND GRIP PRESSURE (kg.) AND BODY WEIGHT (log kg.) FOR MEN

\begin{tabular}{|c|c|c|c|c|c|c|c|c|c|c|c|}
\hline \multirow{2}{*}{$\begin{array}{c}\text { Hand Grip } \\
\text { Pressure }\end{array}$} & \multicolumn{10}{|c|}{ Log Weight } & \multirow[b]{2}{*}{ Total } \\
\hline & $1 \cdot 6000$ & $1 \cdot 6400$ & $1 \cdot 6800$ & $1 \cdot 7200$ & $1 \cdot 7600$ & $1 \cdot 8000$ & $1 \cdot 8400$ & $1 \cdot 8800$ & $1 \cdot 9200$ & $1 \cdot 9600-1 \cdot 9999$ & \\
\hline $22-$ & & & & 1 & & & & & & & 1 \\
\hline $26-$ & & & 2 & 3 & 3 & 1 & & & & & 9 \\
\hline $30-$ & 1 & 4 & 11 & 11 & 12 & 7 & 4 & & & & 50 \\
\hline 34 & & 2 & 12 & 10 & 20 & 18 & 13 & 1 & & & 76 \\
\hline $38-$ & & 3 & 14 & 16 & 25 & 25 & 22 & 12 & & & 117 \\
\hline $42-$ & & & 1 & 6 & 12 & 13 & 17 & 8 & 5 & & 62 \\
\hline 46 & & & & 4 & 10 & 9 & 9 & 4 & 2 & 1 & 39 \\
\hline $50-$ & & & & & & 1 & 3 & 2 & 1 & & 7 \\
\hline $54-57$ & & & & & & & & & 1 & & 1 \\
\hline Total & 1 & 9 & 40 & 51 & 82 & 74 & 68 & 27 & 9 & 1 & 362 \\
\hline
\end{tabular}

TABLE III

DISTRIBUTION OF RIGHT HAND GRIP PRESSURE (kg.) AND BODY WEIGHT (log kg.) FOR WOMEN

\begin{tabular}{|c|c|c|c|c|c|c|c|c|}
\hline \multirow{2}{*}{$\begin{array}{c}\text { Hand Grip } \\
\text { Pressure }\end{array}$} & \multicolumn{7}{|c|}{ Log Weight } & \multirow[b]{2}{*}{ Total } \\
\hline & $1 \cdot 5200$ & $1 \cdot 6000$ & $1 \cdot 6800$ & $1 \cdot 7600$ & $1 \cdot 8400$ & $1 \cdot 9200-$ & $2 \cdot 0000-2 \cdot 7999$ & \\
\hline $20-$ & & & 2 & & & & & 2 \\
\hline $22-$ & 1 & 1 & 4 & 4 & 2 & & & 12 \\
\hline 24 & & 7 & 8 & 7 & 4 & 2 & 1 & 29 \\
\hline 26 & 3 & 7 & 19 & 16 & 12 & 2 & & 59 \\
\hline $28-$ & 2 & 11 & 24 & 29 & 20 & 4 & & 90 \\
\hline 30 & 1 & 4 & 11 & 34 & 24 & 5 & & 79 \\
\hline $32-$ & & 3 & 9 & 22 & 17 & 9 & 2 & 62 \\
\hline 34 & & 1 & 7 & 16 & 11 & 3 & & 38 \\
\hline $36-$ & & & 4 & 7 & 4 & & & 15 \\
\hline $38-$ & & & 1 & 3 & 2 & & & 6 \\
\hline $40-41$ & & & & 2 & & & & 2 \\
\hline Total & 7 & 34 & 89 & 140 & 96 & 25 & 3 & 394 \\
\hline
\end{tabular}


The data of Table II indicate that the regression of right hand grip pressure on body weight for men is linear as follows:

\begin{tabular}{|c|c|c|c|}
\hline $\begin{array}{l}\text { Source of } \\
\text { Variation }\end{array}$ & $\begin{array}{c}\begin{array}{c}\text { Degrees } \\
\text { of } \\
\text { Freedom }\end{array} \\
\end{array}$ & $\begin{array}{l}\text { Sum of } \\
\text { Squares }\end{array}$ & $\begin{array}{l}\text { Mean } \\
\text { Square }\end{array}$ \\
\hline $\begin{array}{llll}\text { Total } & \ldots & \ldots & \ldots \\
\text { Weights } & \ldots & \ldots & \ldots\end{array}$ & $\begin{array}{r}361 \\
9\end{array}$ & $\begin{array}{r}10612 \cdot 6 \\
2150.9\end{array}$ & \\
\hline $\begin{array}{cr}\text { Individuals in } & \text { Weight } \\
\text { Groups (error) } & . .\end{array}$ & 352 & $8461 \cdot 7$ & 24.04 \\
\hline $\begin{array}{l}\text { Weights } \\
\text { Linear Deviation }\end{array}$ & $\begin{array}{l}9 \\
1\end{array}$ & $\begin{array}{l}2150 \cdot 9 \\
1968 \cdot 7\end{array}$ & \\
\hline $\begin{array}{c}\text { Deviations from } \\
\text { Regression } \quad \ldots \\
\text {. }\end{array}$ & 8 & $182 \cdot 2$ & $22 \cdot 77$ \\
\hline
\end{tabular}

The regression involving these two variables is linear also for the left hand of men. This linearity of regression is doubtless related to the absence of obese men in this study. None were 25 per cent. or more over ideal weight as estimated from Anderson's nomogram (1948). Of the 394 women, 108 were 25 per cent. or more over ideal weight. It may be as expected, therefore, that women show a significant departure from linear regression of hand grip pressures on body weight as shown in Table IV (below).

The regression of hand grip pressure $(Y)$ on log weight $\left(X_{1}\right)$ and age $\left(X_{2}\right)$ is assumed to be for men of the form

$$
\hat{Y}=a X_{1}+b X_{2}+C
$$

and for women of the form

$$
\hat{Y}=a X_{1}+b X_{1}^{2}+c X_{2}+d
$$

where $a, b, c$, and $d$ are constants determined by the method of least squares.
The data give regressions whose equations are:

$$
\begin{array}{ccc}
\text { MeN } & \text { Right } & Y=32.945028 X_{1}-0.32700776 X_{2} \\
& & +3.953282 \\
& \text { Left } & Y= \\
& & -0.83 .90594 X_{1}-0.31281065 X_{2} \\
\text { WOMEN } & \text { Right } & Y=146.988935 X_{1}-39.728208 X_{1}^{2} \\
& & -0.1990848 X_{2}-91.23304 \\
& \text { Left } & Y=179.298176 X_{1}-48.448874 X_{1}^{2} \\
& & -0.2181515 X_{2}-121.48802
\end{array}
$$

where $Y=$ hand grip pressure (kg.), $X_{1}=$ body weight ( $\log \mathrm{kg}$.), and $X_{2}=$ age (completed years).

The standard deviations of the observed values of hand grip pressures from the regressions given above are:

MEN Right $S_{Y .12}=4 \cdot 354327 \mathrm{~kg}$.

WOMEN Right $S_{Y .123}=3.227979 \mathrm{~kg}$.

Left $S_{Y .123}=3 \cdot 126425 \mathrm{~kg}$.

The coefficients of $X_{1}$ and $X_{2}$ for men and of $X_{1}, X_{1}^{2}$, and $X_{2}$ for women are highly significantly different from zero $(P<0.01$ for all coefficients).

\begin{tabular}{|c|c|c|c|c|c|}
\hline Hand & Source of Variation & & $\begin{array}{l}\text { Degrees of } \\
\text { Freedom }\end{array}$ & $\begin{array}{l}\text { Sum of } \\
\text { Squares }\end{array}$ & Mean Square \\
\hline \multirow{2}{*}{$\begin{array}{c}\text { Right } \\
(F=8 \cdot 37)\end{array}$} & $\begin{array}{l}\text { Deviations from linear regression } \\
\text { Deviations from curved regression }\end{array}$ & $\begin{array}{l}. \\
\therefore\end{array}$ & $\begin{array}{l}392 \\
391\end{array}$ & $\begin{array}{l}4829 \cdot 2 \\
4728 \cdot 0\end{array}$ & $12 \cdot 09$ \\
\hline & Curvilinearity of regression & . & 1 & $101 \cdot 2$ & $101 \cdot 20$ \\
\hline \multirow{2}{*}{$\begin{array}{c}\text { Left } \\
(F=12 \cdot 38)\end{array}$} & $\begin{array}{l}\text { Deviations from linear regression } \\
\text { Deviations from curved regression }\end{array}$ & 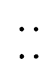 & $\begin{array}{l}392 \\
391\end{array}$ & $\begin{array}{l}4755 \cdot 6 \\
4609 \cdot 6\end{array}$ & $11 \cdot 79$ \\
\hline & Curvilinearity of regression & . & 1 & $146 \cdot 0$ & $146 \cdot 00$ \\
\hline
\end{tabular}

Tables V to VIII show for men and women the means and first and ninety-ninth percentiles of hand grip pressure in terms of body weight and age.

The "multipliers" used in the significance tests and to calculate the confidence interval estimates on the regressions of hand grip pressures are:

$\begin{array}{ll}\text { MeN } & c_{11}=0.63690338 \\ & c_{22}=0.000057420133 \\ \text { WOMEN } & c_{12}=0.00042432324 \\ & c_{11}=277.431399 \\ & c_{22}=21 \cdot 7472025 \\ & c_{33}=0.000059668217 \\ & c_{12}=-77.6105748 \\ & c_{13}=0.0091223216 \\ & c_{23}=-0.0021262042\end{array}$

TABLE IV

FINDINGS IN WOMEN 
TABLE V

MEANS AND PERCENTILES OF RIGHT HAND GRIP PRESSURE (kg.) FOR MEN, IN TERMS OF BODY WEIGHT AND AGE

\begin{tabular}{|c|c|c|c|c|c|c|c|c|c|c|c|}
\hline \multirow{2}{*}{$\begin{array}{l}\text { Body Weight } \\
\text { (log kg.) }\end{array}$} & \multirow{2}{*}{$\begin{array}{l}\text { Percentile } \\
\text { and } \\
\text { Mean }\end{array}$} & \multicolumn{10}{|c|}{ Age (yrs) } \\
\hline & & 60 & $63-$ & $66-$ & 69- & $72-$ & 75 & 78- & 81- & 84 & $87-89$ \\
\hline 1.5800 & $\begin{array}{l}\text { 1st } \\
\text { Mean } \\
\text { 99th }\end{array}$ & $\begin{array}{l}34 \cdot 4 \\
36 \cdot 6 \\
38 \cdot 7\end{array}$ & $\begin{array}{l}33 \cdot 5 \\
35.6 \\
37.6\end{array}$ & $\begin{array}{l}32 \cdot 6 \\
34 \cdot 6 \\
36.5\end{array}$ & $\begin{array}{l}31 \cdot 7 \\
33 \cdot 6 \\
35 \cdot 5\end{array}$ & $\begin{array}{l}30 \cdot 8 \\
32 \cdot 6 \\
34 \cdot 5\end{array}$ & $\begin{array}{l}29.8 \\
31.6 \\
33.5\end{array}$ & $\begin{array}{l}28 \cdot 7 \\
30.7 \\
32 \cdot 6\end{array}$ & $\begin{array}{l}27 \cdot 7 \\
29.7 \\
31.7\end{array}$ & $\begin{array}{l}26.6 \\
28.7 \\
30.8\end{array}$ & $\begin{array}{l}25.5 \\
27.7 \\
30.0\end{array}$ \\
\hline 1.6200 & $\begin{array}{l}\text { st } \\
\text { Mean } \\
\text { 99th }\end{array}$ & $\begin{array}{l}36 \cdot 0 \\
37 \cdot 9 \\
39 \cdot 7\end{array}$ & $\begin{array}{l}35 \cdot 2 \\
36 \cdot 9 \\
38 \cdot 6\end{array}$ & $\begin{array}{l}34 \cdot 3 \\
35 \cdot 9 \\
37 \cdot 5\end{array}$ & $\begin{array}{l}33 \cdot 4 \\
34 \cdot 9 \\
36 \cdot 5\end{array}$ & $\begin{array}{l}32 \cdot 4 \\
33 \cdot 9 \\
35 \cdot 5\end{array}$ & $\begin{array}{l}31.4 \\
33.0 \\
34.5\end{array}$ & $\begin{array}{l}30 \cdot 4 \\
32 \cdot 0 \\
33 \cdot 6\end{array}$ & $\begin{array}{l}29 \cdot 3 \\
31 \cdot 0 \\
32 \cdot 7\end{array}$ & $\begin{array}{l}28 \cdot 2 \\
30 \cdot 0 \\
31 \cdot 9\end{array}$ & $\begin{array}{l}27 \cdot 0 \\
29 \cdot 0 \\
31 \cdot 0\end{array}$ \\
\hline 1.6600 & $\begin{array}{l}\text { 1st } \\
\text { Mean } \\
\text { 99th }\end{array}$ & $\begin{array}{l}37 \cdot 6 \\
39 \cdot 2 \\
40 \cdot 8\end{array}$ & $\begin{array}{l}36 \cdot 8 \\
38.2 \\
39 \cdot 6\end{array}$ & $\begin{array}{l}35 \cdot 9 \\
37 \cdot 2 \\
38 \cdot 5\end{array}$ & $\begin{array}{l}35.0 \\
36.2 \\
37.5\end{array}$ & $\begin{array}{l}34 \cdot 1 \\
35 \cdot 3 \\
36 \cdot 5\end{array}$ & $\begin{array}{l}33 \cdot 0 \\
34 \cdot 3 \\
35 \cdot 5\end{array}$ & $\begin{array}{l}32 \cdot 0 \\
33 \cdot 3 \\
34 \cdot 6\end{array}$ & $\begin{array}{l}30 \cdot 9 \\
32 \cdot 3 \\
33 \cdot 8\end{array}$ & $\begin{array}{l}29 \cdot 7 \\
31 \cdot 3 \\
33 \cdot 0\end{array}$ & $\begin{array}{l}28.6 \\
30.4 \\
32.2\end{array}$ \\
\hline $1 \cdot 7000$ & $\begin{array}{l}\text { 1st } \\
\text { Mean } \\
\text { 99th }\end{array}$ & $\begin{array}{l}39 \cdot 2 \\
40 \cdot 5 \\
41 \cdot 8\end{array}$ & $\begin{array}{l}38 \cdot 3 \\
39 \cdot 5 \\
40 \cdot 7\end{array}$ & $\begin{array}{l}37 \cdot 5 \\
38 \cdot 5 \\
39 \cdot 6\end{array}$ & $\begin{array}{l}36 \cdot 6 \\
37 \cdot 6 \\
38.5\end{array}$ & $\begin{array}{l}35 \cdot 6 \\
36 \cdot 6 \\
37 \cdot 5\end{array}$ & $\begin{array}{l}34 \cdot 6 \\
35.6 \\
36.6\end{array}$ & $\begin{array}{l}33 \cdot 5 \\
34.6 \\
35.7\end{array}$ & $\begin{array}{l}32.4 \\
33 \cdot 6 \\
34.9\end{array}$ & $\begin{array}{l}31 \cdot 3 \\
32 \cdot 7 \\
34 \cdot 1\end{array}$ & $\begin{array}{l}30 \cdot 1 \\
31 \cdot 7 \\
33 \cdot 3\end{array}$ \\
\hline 1.7400 & $\begin{array}{l}\text { st } \\
\text { Mean } \\
\text { 99th }\end{array}$ & $\begin{array}{l}40 \cdot 6 \\
41 \cdot 8 \\
43 \cdot 0\end{array}$ & $\begin{array}{l}39.8 \\
40.8 \\
41.8\end{array}$ & $\begin{array}{l}39 \cdot 0 \\
39 \cdot 9 \\
40 \cdot 7\end{array}$ & $\begin{array}{l}38 \cdot 2 \\
38 \cdot 9 \\
39 \cdot 6\end{array}$ & $\begin{array}{l}37 \cdot 2 \\
37.9 \\
38 \cdot 6\end{array}$ & $\begin{array}{l}36 \cdot 1 \\
36 \cdot 9 \\
37 \cdot 7\end{array}$ & $\begin{array}{l}35.0 \\
35.9 \\
36.8\end{array}$ & $\begin{array}{l}33 \cdot 9 \\
35 \cdot 0 \\
36 \cdot 0\end{array}$ & $\begin{array}{l}32 \cdot 7 \\
34 \cdot 0 \\
35 \cdot 3\end{array}$ & $\begin{array}{l}31 \cdot 5 \\
33 \cdot 0 \\
34.5\end{array}$ \\
\hline 1.7800 & $\begin{array}{l}\text { st } \\
\text { Mean } \\
\text { 99th }\end{array}$ & $\begin{array}{l}42 \cdot 0 \\
43 \cdot 1 \\
44 \cdot 2\end{array}$ & $\begin{array}{l}41 \cdot 3 \\
42 \cdot 2 \\
43 \cdot 1\end{array}$ & $\begin{array}{l}40.4 \\
41.2 \\
41.9\end{array}$ & $\begin{array}{l}39 \cdot 6 \\
40 \cdot 2 \\
40 \cdot 8\end{array}$ & $\begin{array}{l}38 \cdot 6 \\
39 \cdot 2 \\
39 \cdot 8\end{array}$ & $\begin{array}{l}37 \cdot 5 \\
38.2 \\
38.9\end{array}$ & $\begin{array}{l}36 \cdot 4 \\
37 \cdot 3 \\
38 \cdot 1\end{array}$ & $\begin{array}{l}35 \cdot 2 \\
36 \cdot 3 \\
37 \cdot 3\end{array}$ & $\begin{array}{l}34 \cdot 0 \\
35 \cdot 3 \\
36 \cdot 6\end{array}$ & $\begin{array}{l}32 \cdot 8 \\
34 \cdot 3 \\
35 \cdot 8\end{array}$ \\
\hline 1.8200 & $\begin{array}{l}\text { Ist } \\
\text { Mean } \\
\text { 99th }\end{array}$ & $\begin{array}{l}43 \cdot 3 \\
44 \cdot 5 \\
45 \cdot 6\end{array}$ & $\begin{array}{l}42 \cdot 5 \\
43 \cdot 5 \\
44.4\end{array}$ & $\begin{array}{l}41 \cdot 7 \\
42.5 \\
43.3\end{array}$ & $\begin{array}{l}40 \cdot 8 \\
41 \cdot 5 \\
42 \cdot 2\end{array}$ & $\begin{array}{l}39 \cdot 8 \\
40 \cdot 5 \\
41 \cdot 2\end{array}$ & $\begin{array}{l}38 \cdot 8 \\
39 \cdot 6 \\
40 \cdot 4\end{array}$ & $\begin{array}{l}37 \cdot 6 \\
38 \cdot 6 \\
39 \cdot 5\end{array}$ & $\begin{array}{l}36 \cdot 5 \\
37 \cdot 5 \\
38 \cdot 7\end{array}$ & $\begin{array}{l}35 \cdot 3 \\
36 \cdot 6 \\
38 \cdot 0\end{array}$ & $\begin{array}{l}34 \cdot 1 \\
35 \cdot 6 \\
37 \cdot 2\end{array}$ \\
\hline 1.8600 & $\begin{array}{l}\text { st } \\
\text { Mean } \\
\text { 99th }\end{array}$ & $\begin{array}{l}44 \cdot 5 \\
45.8 \\
47 \cdot 1\end{array}$ & $\begin{array}{l}43 \cdot 7 \\
44.8 \\
45.9\end{array}$ & $\begin{array}{l}42 \cdot 8 \\
43.8 \\
44.8\end{array}$ & $\begin{array}{l}41 \cdot 9 \\
42 \cdot 8 \\
43 \cdot 8\end{array}$ & $\begin{array}{l}40 \cdot 9 \\
41 \cdot 8 \\
42 \cdot 8\end{array}$ & $\begin{array}{l}39 \cdot 8 \\
40.9 \\
41.9\end{array}$ & $\begin{array}{l}38.7 \\
39.9 \\
41 \cdot 1\end{array}$ & $\begin{array}{l}37 \cdot 6 \\
38.9 \\
40 \cdot 2\end{array}$ & $\begin{array}{l}36.4 \\
37.9 \\
39.4\end{array}$ & $\begin{array}{l}35 \cdot 2 \\
37 \cdot 0 \\
38 \cdot 7\end{array}$ \\
\hline 1.9000 & $\begin{array}{l}\text { 1st } \\
\text { Mean } \\
\text { 99th }\end{array}$ & $\begin{array}{l}45 \cdot 6 \\
47 \cdot 1 \\
48 \cdot 6\end{array}$ & $\begin{array}{l}44 \cdot 7 \\
46.1 \\
47.5\end{array}$ & $\begin{array}{l}43 \cdot 8 \\
45.1 \\
46 \cdot 4\end{array}$ & $\begin{array}{l}42 \cdot 9 \\
44 \cdot 1 \\
45 \cdot 4\end{array}$ & $\begin{array}{l}41 \cdot 9 \\
43 \cdot 2 \\
44 \cdot 4\end{array}$ & $\begin{array}{l}40 \cdot 9 \\
42 \cdot 2 \\
43 \cdot 5\end{array}$ & $\begin{array}{l}39 \cdot 8 \\
41.2 \\
42 \cdot 6\end{array}$ & $\begin{array}{l}38 \cdot 7 \\
40 \cdot 2 \\
41 \cdot 8\end{array}$ & $\begin{array}{l}37 \cdot 5 \\
39 \cdot 2 \\
41 \cdot 0\end{array}$ & $\begin{array}{l}36 \cdot 4 \\
38 \cdot 3 \\
40 \cdot 2\end{array}$ \\
\hline $\begin{array}{c}1.9400 \\
-1.9799\end{array}$ & $\begin{array}{l}\text { 1st } \\
\text { Mean } \\
\text { M9th }\end{array}$ & $\begin{array}{l}46 \cdot 6 \\
48.4 \\
50 \cdot 2\end{array}$ & $\begin{array}{l}45.8 \\
47.4 \\
49.1\end{array}$ & $\begin{array}{l}44 \cdot 8 \\
46.4 \\
48 \cdot 1\end{array}$ & $\begin{array}{l}43.9 \\
45.5 \\
47.1\end{array}$ & $\begin{array}{l}42 \cdot 9 \\
44 \cdot 5 \\
46 \cdot 1\end{array}$ & $\begin{array}{l}41 \cdot 9 \\
43 \cdot 5 \\
45 \cdot 1\end{array}$ & $\begin{array}{l}40 \cdot 8 \\
42 \cdot 5 \\
44 \cdot 2\end{array}$ & $\begin{array}{l}39 \cdot 7 \\
41.6 \\
43.4\end{array}$ & $\begin{array}{l}38.6 \\
40.6 \\
42.5\end{array}$ & $\begin{array}{l}37 \cdot 5 \\
39 \cdot 6 \\
41 \cdot 7\end{array}$ \\
\hline
\end{tabular}

TABLE VI

MEANS AND PERCENTILES OF LEFT HAND GRIP PRESSURE (kg.) FOR MEN, IN TERMS OF BODY WEIGHT AND AGE

\begin{tabular}{|c|c|c|c|c|c|c|c|c|c|c|c|}
\hline \multirow{2}{*}{$\begin{array}{l}\text { Body Weight } \\
\text { (log kg.) }\end{array}$} & \multirow{2}{*}{$\begin{array}{l}\text { Percentile } \\
\text { and } \\
\text { Mean }\end{array}$} & \multicolumn{10}{|c|}{ Age (yrs) } \\
\hline & & 60 & 63- & 66- & 69- & 72 & 75 & 78 & 81- & 84 & $87-89$ \\
\hline 1.5800 & $\begin{array}{l}\text { 1st } \\
\text { Mean } \\
\text { 99th }\end{array}$ & $\begin{array}{l}32 \cdot 1 \\
34 \cdot 2 \\
36 \cdot 3\end{array}$ & $\begin{array}{l}31 \cdot 3 \\
33 \cdot 3 \\
35 \cdot 2\end{array}$ & $\begin{array}{l}30 \cdot 4 \\
32 \cdot 3 \\
34 \cdot 2\end{array}$ & $\begin{array}{l}29 \cdot 5 \\
31 \cdot 4 \\
33 \cdot 2\end{array}$ & $\begin{array}{l}28 \cdot 6 \\
30 \cdot 4 \\
32 \cdot 2\end{array}$ & $\begin{array}{l}27 \cdot 7 \\
29 \cdot 5 \\
31 \cdot 3\end{array}$ & $\begin{array}{l}26 \cdot 7 \\
28 \cdot 6 \\
30 \cdot 4\end{array}$ & $\begin{array}{l}25 \cdot 7 \\
27 \cdot 6 \\
29 \cdot 5\end{array}$ & $\begin{array}{l}24 \cdot 6 \\
26.7 \\
28.7\end{array}$ & $\begin{array}{l}23.6 \\
25.7 \\
27.9\end{array}$ \\
\hline 1.6200 & $\begin{array}{l}\text { 1st } \\
\text { Mean } \\
\text { 99th }\end{array}$ & $\begin{array}{l}33.8 \\
35.5 \\
37.3\end{array}$ & $\begin{array}{l}33 \cdot 0 \\
34 \cdot 6 \\
36 \cdot 2\end{array}$ & $\begin{array}{l}32 \cdot 1 \\
33 \cdot 7 \\
35 \cdot 2\end{array}$ & $\begin{array}{l}31 \cdot 2 \\
32 \cdot 7 \\
34 \cdot 2\end{array}$ & $\begin{array}{l}30 \cdot 3 \\
31 \cdot 8 \\
33 \cdot 3\end{array}$ & $\begin{array}{l}29 \cdot 3 \\
30 \cdot 8 \\
32 \cdot 3\end{array}$ & $\begin{array}{l}28.4 \\
29.9 \\
31 \cdot 5\end{array}$ & $\begin{array}{l}27 \cdot 3 \\
29 \cdot 0 \\
30 \cdot 6\end{array}$ & $\begin{array}{l}26 \cdot 3 \\
28 \cdot 0 \\
29 \cdot 8\end{array}$ & $\begin{array}{l}25.2 \\
27.1 \\
29.0\end{array}$ \\
\hline 1.6600 & $\begin{array}{l}\text { st } \\
\text { Mean } \\
\text { 99th }\end{array}$ & $\begin{array}{l}35.4 \\
36.9 \\
38.4\end{array}$ & $\begin{array}{l}34 \cdot 6 \\
36 \cdot 0 \\
37 \cdot 4\end{array}$ & $\begin{array}{l}33.7 \\
35.0 \\
36.3\end{array}$ & $\begin{array}{l}32 \cdot 9 \\
34 \cdot 1 \\
35 \cdot 3\end{array}$ & $\begin{array}{l}32 \cdot 0 \\
33 \cdot 1 \\
34 \cdot 3\end{array}$ & $\begin{array}{l}31 \cdot 0 \\
32 \cdot 2 \\
33 \cdot 4\end{array}$ & $\begin{array}{l}30 \cdot 0 \\
31 \cdot 3 \\
32 \cdot 6\end{array}$ & $\begin{array}{l}29 \cdot 0 \\
30 \cdot 3 \\
31 \cdot 7\end{array}$ & $\begin{array}{l}27.9 \\
29.4 \\
30.9\end{array}$ & $\begin{array}{l}26.7 \\
28.4 \\
30.2\end{array}$ \\
\hline $1 \cdot 7000$ & $\begin{array}{l}\text { 1st } \\
\text { Mean } \\
\text { 99th }\end{array}$ & $\begin{array}{l}37.0 \\
38.3 \\
39.6 \\
\end{array}$ & $\begin{array}{l}36 \cdot 2 \\
37 \cdot 3 \\
38 \cdot 4\end{array}$ & $\begin{array}{l}35.4 \\
36.4 \\
37.4\end{array}$ & $\begin{array}{l}34 \cdot 5 \\
35 \cdot 4 \\
36 \cdot 3\end{array}$ & $\begin{array}{l}33 \cdot 6 \\
34 \cdot 5 \\
35 \cdot 4\end{array}$ & $\begin{array}{l}32 \cdot 6 \\
33 \cdot 6 \\
34 \cdot 5\end{array}$ & $\begin{array}{l}31 \cdot 6 \\
32 \cdot 6 \\
33 \cdot 6\end{array}$ & $\begin{array}{l}30.5 \\
31.7 \\
32.9\end{array}$ & $\begin{array}{l}29.4 \\
30.7 \\
32.1\end{array}$ & $\begin{array}{l}28.2 \\
29.8 \\
31.4\end{array}$ \\
\hline 1.7400 & $\begin{array}{l}\text { 1st } \\
\text { Mean } \\
\text { 99th }\end{array}$ & $\begin{array}{l}38 \cdot 5 \\
39 \cdot 6 \\
40 \cdot 7\end{array}$ & $\begin{array}{l}37 \cdot 7 \\
38 \cdot 7 \\
39 \cdot 6\end{array}$ & $\begin{array}{l}36 \cdot 9 \\
37 \cdot 9 \\
38.5\end{array}$ & $\begin{array}{l}36 \cdot 1 \\
36 \cdot 8 \\
37.5\end{array}$ & $\begin{array}{l}35 \cdot 2 \\
35 \cdot 9 \\
36 \cdot 5\end{array}$ & $\begin{array}{l}34 \cdot 2 \\
34.9 \\
35.7\end{array}$ & $\begin{array}{l}33 \cdot 1 \\
34 \cdot 0 \\
34 \cdot 9\end{array}$ & $\begin{array}{l}32 \cdot 0 \\
33.0 \\
34 \cdot 1\end{array}$ & $\begin{array}{l}30 \cdot 9 \\
32 \cdot 1 \\
33 \cdot 3\end{array}$ & $\begin{array}{l}29 \cdot 7 \\
31.2 \\
32.6\end{array}$ \\
\hline
\end{tabular}


Table VI, continued

\begin{tabular}{|c|c|c|c|c|c|c|c|c|c|c|c|}
\hline \multirow{2}{*}{$\begin{array}{l}\text { Body Weight } \\
\text { (log kg.) }\end{array}$} & \multirow{2}{*}{$\begin{array}{c}\text { Percentile } \\
\text { and } \\
\text { Mean }\end{array}$} & \multicolumn{10}{|c|}{ Age (yrs) } \\
\hline & & $60-$ & $63-$ & $66-$ & 69- & $72-$ & $75-$ & $78-$ & $81-$ & $84-$ & $87-89$ \\
\hline $1 \cdot 7800$ & $\begin{array}{l}\text { 1st } \\
\text { Mean } \\
\text { 99th }\end{array}$ & $\begin{array}{l}39 \cdot 9 \\
41 \cdot 0 \\
42 \cdot 0\end{array}$ & $\begin{array}{l}39 \cdot 2 \\
40 \cdot 0 \\
40 \cdot 9\end{array}$ & $\begin{array}{l}38 \cdot 4 \\
39 \cdot 1 \\
39 \cdot 8\end{array}$ & $\begin{array}{l}37 \cdot 5 \\
38 \cdot 1 \\
38 \cdot 7\end{array}$ & $\begin{array}{l}36 \cdot 6 \\
37 \cdot 2 \\
37 \cdot 8\end{array}$ & $\begin{array}{l}35 \cdot 6 \\
36 \cdot 3 \\
36 \cdot 9\end{array}$ & $\begin{array}{l}34 \cdot 5 \\
35 \cdot 3 \\
36 \cdot 1\end{array}$ & $\begin{array}{l}33 \cdot 4 \\
34 \cdot 4 \\
35 \cdot 4\end{array}$ & $\begin{array}{l}32 \cdot 2 \\
33 \cdot 5 \\
34 \cdot 7\end{array}$ & $\begin{array}{l}31 \cdot 0 \\
32 \cdot 5 \\
34 \cdot 1\end{array}$ \\
\hline $1 \cdot 8200$ & $\begin{array}{l}\text { 1st } \\
\text { Mean } \\
\text { 99th }\end{array}$ & $\begin{array}{l}41 \cdot 2 \\
42 \cdot 3 \\
43 \cdot 4\end{array}$ & $\begin{array}{l}40 \cdot 5 \\
41 \cdot 4 \\
42 \cdot 3\end{array}$ & $\begin{array}{l}39 \cdot 7 \\
40 \cdot 4 \\
41 \cdot 2\end{array}$ & $\begin{array}{l}38 \cdot 8 \\
39 \cdot 5 \\
40 \cdot 2\end{array}$ & $\begin{array}{l}37 \cdot 9 \\
38 \cdot 6 \\
39 \cdot 3\end{array}$ & $\begin{array}{l}36 \cdot 9 \\
37 \cdot 6 \\
38 \cdot 4\end{array}$ & $\begin{array}{l}35 \cdot 8 \\
36 \cdot 7 \\
37 \cdot 6\end{array}$ & $\begin{array}{l}34 \cdot 7 \\
35 \cdot 7 \\
36 \cdot 8\end{array}$ & $\begin{array}{l}33 \cdot 5 \\
34 \cdot 8 \\
36 \cdot 1\end{array}$ & $\begin{array}{l}32 \cdot 3 \\
33 \cdot 9 \\
35 \cdot 4\end{array}$ \\
\hline $1 \cdot 8600$ & $\begin{array}{l}\text { 1st } \\
\text { Mean } \\
99 \text { th }\end{array}$ & $\begin{array}{l}42 \cdot 5 \\
43 \cdot 7 \\
44 \cdot 9\end{array}$ & $\begin{array}{l}41 \cdot 7 \\
42 \cdot 7 \\
43 \cdot 8\end{array}$ & $\begin{array}{l}40 \cdot 8 \\
41 \cdot 8 \\
42 \cdot 8\end{array}$ & $\begin{array}{l}40 \cdot 0 \\
40 \cdot 9 \\
41 \cdot 8\end{array}$ & $\begin{array}{l}39 \cdot 0 \\
39.9 \\
40.8\end{array}$ & $\begin{array}{l}38 \cdot 0 \\
39 \cdot 0 \\
40 \cdot 0\end{array}$ & $\begin{array}{l}37 \cdot 0 \\
38 \cdot 0 \\
39 \cdot 1\end{array}$ & $\begin{array}{l}35 \cdot 9 \\
37 \cdot 1 \\
38 \cdot 4\end{array}$ & $\begin{array}{l}34 \cdot 7 \\
36 \cdot 2 \\
37 \cdot 6\end{array}$ & $\begin{array}{l}33 \cdot 6 \\
35 \cdot 2 \\
36 \cdot 9\end{array}$ \\
\hline $1 \cdot 9000$ & $\begin{array}{l}\text { 1st } \\
\text { Mean } \\
99 \text { th }\end{array}$ & $\begin{array}{l}43 \cdot 6 \\
45 \cdot 0 \\
46 \cdot 5\end{array}$ & $\begin{array}{l}42 \cdot 8 \\
44 \cdot 1 \\
45 \cdot 4\end{array}$ & $\begin{array}{l}41 \cdot 9 \\
43 \cdot 2 \\
44 \cdot 4\end{array}$ & $\begin{array}{l}41 \cdot 0 \\
42 \cdot 2 \\
43 \cdot 4\end{array}$ & $\begin{array}{l}40 \cdot 1 \\
41 \cdot 3 \\
42 \cdot 5\end{array}$ & $\begin{array}{l}39 \cdot 1 \\
40 \cdot 3 \\
41 \cdot 6\end{array}$ & $\begin{array}{l}38 \cdot 1 \\
39 \cdot 4 \\
40 \cdot 8\end{array}$ & $\begin{array}{l}37 \cdot 0 \\
38 \cdot 5 \\
40 \cdot 0\end{array}$ & $\begin{array}{l}35 \cdot 9 \\
37 \cdot 5 \\
39 \cdot 2\end{array}$ & $\begin{array}{l}34 \cdot 8 \\
36 \cdot 6 \\
38 \cdot 4\end{array}$ \\
\hline $\begin{array}{l}1 \cdot 9400 \\
-1 \cdot 9799\end{array}$ & $\begin{array}{l}\text { 1st } \\
\text { Mean } \\
\text { 99th }\end{array}$ & $\begin{array}{l}44 \cdot 7 \\
46 \cdot 4 \\
48 \cdot 1\end{array}$ & $\begin{array}{l}43 \cdot 8 \\
45 \cdot 5 \\
47 \cdot 1\end{array}$ & $\begin{array}{l}43 \cdot 0 \\
44 \cdot 5 \\
46 \cdot 1\end{array}$ & $\begin{array}{l}42 \cdot 1 \\
43 \cdot 6 \\
45 \cdot 1\end{array}$ & $\begin{array}{l}41 \cdot 1 \\
42 \cdot 6 \\
44 \cdot 1\end{array}$ & $\begin{array}{l}40 \cdot 1 \\
41 \cdot 7 \\
43 \cdot 3\end{array}$ & $\begin{array}{l}39 \cdot 1 \\
40 \cdot 8 \\
42 \cdot 4\end{array}$ & $\begin{array}{l}38 \cdot 1 \\
39 \cdot 8 \\
41 \cdot 6\end{array}$ & $\begin{array}{l}37.0 \\
38.9 \\
40.8\end{array}$ & $\begin{array}{l}35 \cdot 9 \\
37 \cdot 9 \\
40 \cdot 0\end{array}$ \\
\hline
\end{tabular}

TABLE VII

MEANS AND PERCENTILES OF RIGHT HAND GRIP PRESSURE (kg.) FOR WOMEN, IN TERMS OF BODY WEIGHT AND AGE

\begin{tabular}{|c|c|c|c|c|c|c|c|c|c|c|c|}
\hline \multirow{2}{*}{$\begin{array}{l}\text { Body Weight } \\
\text { (log kg.) }\end{array}$} & \multirow{2}{*}{$\begin{array}{l}\text { Percentile } \\
\text { and } \\
\text { Mean }\end{array}$} & \multicolumn{10}{|c|}{ Age (yrs) } \\
\hline & & 60 & $63-$ & $66-$ & 69 & 72 & 75 & 78 & $81-$ & 84 & $87-89$ \\
\hline $1 \cdot 5400$ & $\begin{array}{l}\text { 1st } \\
\text { Mean } \\
\text { 99th }\end{array}$ & $\begin{array}{l}26 \cdot 8 \\
29 \cdot 1 \\
31 \cdot 5\end{array}$ & $\begin{array}{l}26 \cdot 3 \\
28 \cdot 5 \\
30 \cdot 8\end{array}$ & $\begin{array}{l}25 \cdot 8 \\
27.9 \\
30 \cdot 1\end{array}$ & $\begin{array}{l}25 \cdot 2 \\
27 \cdot 3 \\
29 \cdot 5\end{array}$ & $\begin{array}{l}24 \cdot 7 \\
26 \cdot 7 \\
28 \cdot 8\end{array}$ & $\begin{array}{l}24 \cdot 1 \\
26 \cdot 2 \\
28 \cdot 2\end{array}$ & $\begin{array}{l}23.5 \\
25.6 \\
27.6\end{array}$ & $\begin{array}{l}22 \cdot 9 \\
25 \cdot 0 \\
27 \cdot 0\end{array}$ & $\begin{array}{l}22 \cdot 2 \\
24 \cdot 4 \\
26 \cdot 5\end{array}$ & $\begin{array}{l}21 \cdot 6 \\
23 \cdot 8 \\
26 \cdot 0\end{array}$ \\
\hline 1.5800 & $\begin{array}{l}\text { 1st } \\
\text { Mean } \\
\text { 99th }\end{array}$ & $\begin{array}{l}28 \cdot 2 \\
30 \cdot 0 \\
31 \cdot 7\end{array}$ & $\begin{array}{l}27 \cdot 7 \\
29 \cdot 4 \\
31 \cdot 1\end{array}$ & $\begin{array}{l}27 \cdot 2 \\
28 \cdot 8 \\
30 \cdot 4\end{array}$ & $\begin{array}{l}26 \cdot 7 \\
28 \cdot 2 \\
29 \cdot 7\end{array}$ & $\begin{array}{l}26 \cdot 1 \\
27 \cdot 6 \\
29 \cdot 1\end{array}$ & $\begin{array}{l}25 \cdot 5 \\
27 \cdot 0 \\
28 \cdot 5\end{array}$ & $\begin{array}{l}24 \cdot 9 \\
26 \cdot 4 \\
27 \cdot 9\end{array}$ & $\begin{array}{l}24 \cdot 3 \\
25 \cdot 8 \\
27 \cdot 4\end{array}$ & $\begin{array}{l}23 \cdot 6 \\
25 \cdot 2 \\
26 \cdot 8\end{array}$ & $\begin{array}{l}22 \cdot 9 \\
24 \cdot 6 \\
26 \cdot 3\end{array}$ \\
\hline 1.6200 & $\begin{array}{l}\text { 1st } \\
\text { Mean } \\
\text { 99th }\end{array}$ & $\begin{array}{l}29 \cdot 4 \\
30 \cdot 7 \\
32 \cdot 1\end{array}$ & $\begin{array}{l}28 \cdot 9 \\
30 \cdot 1 \\
31 \cdot 3\end{array}$ & $\begin{array}{l}28 \cdot 4 \\
29.5 \\
30 \cdot 7\end{array}$ & $\begin{array}{l}27 \cdot 9 \\
28 \cdot 9 \\
30 \cdot 0\end{array}$ & $\begin{array}{l}27 \cdot 3 \\
28 \cdot 3 \\
29 \cdot 4\end{array}$ & $\begin{array}{l}26 \cdot 7 \\
27 \cdot 7 \\
28 \cdot 8\end{array}$ & $\begin{array}{l}26 \cdot 1 \\
27 \cdot 1 \\
28 \cdot 2\end{array}$ & $\begin{array}{l}25 \cdot 4 \\
26 \cdot 6 \\
27 \cdot 7\end{array}$ & $\begin{array}{l}24 \cdot 7 \\
25 \cdot 9 \\
27 \cdot 2\end{array}$ & $\begin{array}{l}24 \cdot 0 \\
25 \cdot 4 \\
26 \cdot 7\end{array}$ \\
\hline 1.6600 & $\begin{array}{l}\text { 1st } \\
\text { Mean } \\
\text { 99th }\end{array}$ & $\begin{array}{l}30 \cdot 3 \\
31 \cdot 3 \\
32 \cdot 4\end{array}$ & $\begin{array}{l}29 \cdot 8 \\
30 \cdot 7 \\
31 \cdot 6\end{array}$ & $\begin{array}{l}29 \cdot 3 \\
30 \cdot 1 \\
30 \cdot 9\end{array}$ & $\begin{array}{l}28 \cdot 8 \\
29 \cdot 5 \\
30 \cdot 3\end{array}$ & $\begin{array}{l}28 \cdot 2 \\
28 \cdot 9 \\
29 \cdot 7\end{array}$ & $\begin{array}{l}27 \cdot 6 \\
28 \cdot 3 \\
29 \cdot 1\end{array}$ & $\begin{array}{l}26.9 \\
27.7 \\
28.6\end{array}$ & $\begin{array}{l}26 \cdot 2 \\
27 \cdot 1 \\
28 \cdot 1\end{array}$ & $\begin{array}{l}25.5 \\
26.6 \\
27.6\end{array}$ & $\begin{array}{l}24 \cdot 7 \\
26 \cdot 0 \\
27 \cdot 2\end{array}$ \\
\hline $1 \cdot 7000$ & $\begin{array}{l}\text { 1st } \\
\text { Mean } \\
\text { 99th }\end{array}$ & $\begin{array}{l}30.9 \\
31.8 \\
32.7\end{array}$ & $\begin{array}{l}30 \cdot 5 \\
31 \cdot 2 \\
32 \cdot 0\end{array}$ & $\begin{array}{l}30 \cdot 0 \\
30 \cdot 6 \\
31 \cdot 3\end{array}$ & $\begin{array}{l}29 \cdot 4 \\
30 \cdot 0 \\
30 \cdot 6\end{array}$ & $\begin{array}{l}28 \cdot 8 \\
29 \cdot 4 \\
30.0\end{array}$ & $\begin{array}{l}28 \cdot 2 \\
28 \cdot 8 \\
29 \cdot 5\end{array}$ & $\begin{array}{l}27 \cdot 5 \\
28 \cdot 2 \\
29 \cdot 0\end{array}$ & $\begin{array}{l}26 \cdot 7 \\
27.6 \\
28 \cdot 5\end{array}$ & $\begin{array}{l}26 \cdot 0 \\
27 \cdot 0 \\
28 \cdot 1\end{array}$ & $\begin{array}{l}25 \cdot 2 \\
26 \cdot 4 \\
27 \cdot 7\end{array}$ \\
\hline $1 \cdot 7400$ & $\begin{array}{l}\text { 1st } \\
\text { Mean } \\
\text { 99th }\end{array}$ & $\begin{array}{l}31 \cdot 4 \\
32 \cdot 2 \\
32 \cdot 9\end{array}$ & $\begin{array}{l}30 \cdot 9 \\
31 \cdot 6 \\
32 \cdot 2\end{array}$ & $\begin{array}{l}30 \cdot 4 \\
31 \cdot 0 \\
31 \cdot 5\end{array}$ & $\begin{array}{l}29.8 \\
30.4 \\
30.9\end{array}$ & $\begin{array}{l}29 \cdot 2 \\
29 \cdot 8 \\
30 \cdot 4\end{array}$ & $\begin{array}{l}28 \cdot 5 \\
29 \cdot 2 \\
29 \cdot 8\end{array}$ & $\begin{array}{l}27.8 \\
28.6 \\
29.4\end{array}$ & $\begin{array}{l}27 \cdot 0 \\
28 \cdot 0 \\
28 \cdot 9\end{array}$ & $\begin{array}{l}26 \cdot 3 \\
27 \cdot 4 \\
28 \cdot 5\end{array}$ & $\begin{array}{l}25 \cdot 5 \\
26 \cdot 8 \\
28 \cdot 1\end{array}$ \\
\hline $1 \cdot 7800$ & $\begin{array}{l}\text { 1st } \\
\text { Mean } \\
\text { 99th }\end{array}$ & $\begin{array}{l}31 \cdot 6 \\
32 \cdot 4 \\
33 \cdot 1\end{array}$ & $\begin{array}{l}31 \cdot 2 \\
31 \cdot 8 \\
32 \cdot 4\end{array}$ & $\begin{array}{l}30 \cdot 7 \\
31 \cdot 2 \\
31 \cdot 7\end{array}$ & $\begin{array}{l}30 \cdot 1 \\
30 \cdot 6 \\
31 \cdot 1\end{array}$ & $\begin{array}{l}29.4 \\
30.0 \\
30.6\end{array}$ & $\begin{array}{l}28 \cdot 7 \\
29 \cdot 4 \\
30 \cdot 1\end{array}$ & $\begin{array}{l}28 \cdot 0 \\
28 \cdot 8 \\
29 \cdot 6\end{array}$ & $\begin{array}{l}27 \cdot 2 \\
28 \cdot 2 \\
29 \cdot 2\end{array}$ & $\begin{array}{l}26 \cdot 5 \\
27.6 \\
28 \cdot 7\end{array}$ & $\begin{array}{l}25 \cdot 7 \\
27 \cdot 0 \\
28 \cdot 3\end{array}$ \\
\hline 1.8200 & $\begin{array}{l}\text { 1st } \\
\text { Mean } \\
\text { 99th }\end{array}$ & $\begin{array}{l}31 \cdot 8 \\
32 \cdot 5 \\
33 \cdot 2\end{array}$ & $\begin{array}{l}31 \cdot 3 \\
31 \cdot 9 \\
32 \cdot 5\end{array}$ & $\begin{array}{l}30 \cdot 8 \\
31 \cdot 3 \\
31 \cdot 8\end{array}$ & $\begin{array}{l}30 \cdot 1 \\
30 \cdot 7 \\
31 \cdot 2\end{array}$ & $\begin{array}{l}29 \cdot 5 \\
30 \cdot 1 \\
30 \cdot 7\end{array}$ & $\begin{array}{l}28 \cdot 8 \\
29 \cdot 5 \\
30 \cdot 2\end{array}$ & $\begin{array}{l}28 \cdot 0 \\
28 \cdot 9 \\
29 \cdot 8\end{array}$ & $\begin{array}{l}27 \cdot 3 \\
28 \cdot 3 \\
29 \cdot 3\end{array}$ & $\begin{array}{l}26 \cdot 5 \\
27.7 \\
28.9\end{array}$ & $\begin{array}{l}25 \cdot 7 \\
27 \cdot 1 \\
28 \cdot 5\end{array}$ \\
\hline $1 \cdot 8600$ & $\begin{array}{l}\text { 1st } \\
\text { Mean } \\
\text { 99th }\end{array}$ & $\begin{array}{l}31 \cdot 7 \\
32 \cdot 5 \\
33 \cdot 2\end{array}$ & $\begin{array}{l}31 \cdot 2 \\
31 \cdot 8 \\
32 \cdot 5\end{array}$ & $\begin{array}{l}30 \cdot 6 \\
31 \cdot 2 \\
31 \cdot 9\end{array}$ & $\begin{array}{l}30 \cdot 0 \\
30 \cdot 6 \\
31 \cdot 3\end{array}$ & $\begin{array}{l}29 \cdot 3 \\
30 \cdot 1 \\
30 \cdot 8\end{array}$ & $\begin{array}{l}28 \cdot 6 \\
29 \cdot 5 \\
30 \cdot 3\end{array}$ & $\begin{array}{l}27.9 \\
28.9 \\
29.9\end{array}$ & $\begin{array}{l}27 \cdot 1 \\
28 \cdot 3 \\
29 \cdot 4\end{array}$ & $\begin{array}{l}26.4 \\
27.7 \\
29.0\end{array}$ & $\begin{array}{l}25 \cdot 6 \\
27 \cdot 1 \\
28 \cdot 6\end{array}$ \\
\hline 1.9000 & $\begin{array}{l}\text { 1st } \\
\text { Mean } \\
\text { 99th }\end{array}$ & $\begin{array}{l}31 \cdot 3 \\
32 \cdot 3 \\
33 \cdot 3\end{array}$ & $\begin{array}{l}30 \cdot 8 \\
31 \cdot 7 \\
32 \cdot 6\end{array}$ & $\begin{array}{l}30 \cdot 2 \\
31 \cdot 1 \\
32 \cdot 0\end{array}$ & $\begin{array}{l}29 \cdot 6 \\
30.5 \\
31.4\end{array}$ & $\begin{array}{l}28.9 \\
29.9 \\
30.9\end{array}$ & $\begin{array}{l}28 \cdot 2 \\
29 \cdot 3 \\
30 \cdot 4\end{array}$ & $\begin{array}{l}27 \cdot 5 \\
28 \cdot 7 \\
29 \cdot 9\end{array}$ & $\begin{array}{l}26 \cdot 8 \\
28 \cdot 1 \\
29 \cdot 4\end{array}$ & $\begin{array}{l}26.0 \\
27.5 \\
29.0\end{array}$ & $\begin{array}{l}25 \cdot 3 \\
26.9 \\
28.6\end{array}$ \\
\hline $\begin{array}{l}1.9400 \\
-1.9799\end{array}$ & $\begin{array}{l}\text { 1st } \\
\text { Mean } \\
\text { 99th }\end{array}$ & $\begin{array}{l}30 \cdot 6 \\
32 \cdot 0 \\
33 \cdot 4\end{array}$ & $\begin{array}{l}30 \cdot 0 \\
31 \cdot 4 \\
32 \cdot 8\end{array}$ & $\begin{array}{l}29 \cdot 4 \\
30 \cdot 8 \\
32 \cdot 2\end{array}$ & $\begin{array}{l}28 \cdot 8 \\
30 \cdot 2 \\
31 \cdot 6\end{array}$ & $\begin{array}{l}28 \cdot 2 \\
29 \cdot 6 \\
31 \cdot 0\end{array}$ & $\begin{array}{l}27.5 \\
29 \cdot 0 \\
30.5\end{array}$ & $\begin{array}{l}26 \cdot 8 \\
28.4 \\
30.0\end{array}$ & $\begin{array}{l}26 \cdot 1 \\
27 \cdot 8 \\
29 \cdot 5\end{array}$ & $\begin{array}{l}25 \cdot 4 \\
27 \cdot 2 \\
29 \cdot 0\end{array}$ & $\begin{array}{l}24 \cdot 7 \\
26.6 \\
28.6\end{array}$ \\
\hline
\end{tabular}


TABLE VIII

MEANS AND PERCENTILES OF LEFT HAND GRIP PRESSURE (kg.) FOR WOMEN, IN TERMS OF BODY WEIGHT AND AGE

\begin{tabular}{|c|c|c|c|c|c|c|c|c|c|c|c|}
\hline \multirow{2}{*}{$\begin{array}{l}\text { Body Weight } \\
\text { (log kg.) }\end{array}$} & \multirow{2}{*}{$\begin{array}{c}\text { Percentile } \\
\text { and } \\
\text { Mean }\end{array}$} & \multicolumn{10}{|c|}{ Age (yrs) } \\
\hline & & 60 & $63-$ & 66 & 69- & $72-$ & 75 & $78-$ & $81-$ & 84 & $87-89$ \\
\hline $1 \cdot 5400$ & $\begin{array}{l}\text { 1st } \\
\text { Mean } \\
\text { 99th }\end{array}$ & $\begin{array}{l}24 \cdot 6 \\
26 \cdot 9 \\
29 \cdot 1\end{array}$ & $\begin{array}{l}24 \cdot 1 \\
26 \cdot 2 \\
28 \cdot 4\end{array}$ & $\begin{array}{l}23 \cdot 5 \\
25 \cdot 6 \\
27 \cdot 7\end{array}$ & $\begin{array}{l}22 \cdot 9 \\
24 \cdot 9 \\
27 \cdot 0\end{array}$ & $\begin{array}{l}22 \cdot 3 \\
24 \cdot 3 \\
26 \cdot 3\end{array}$ & $\begin{array}{l}21 \cdot 6 \\
23.6 \\
25.6\end{array}$ & $\begin{array}{l}21 \cdot 0 \\
23 \cdot 0 \\
25 \cdot 0\end{array}$ & $\begin{array}{l}20 \cdot 3 \\
22 \cdot 3 \\
24 \cdot 3\end{array}$ & $\begin{array}{l}19 \cdot 6 \\
21 \cdot 7 \\
23 \cdot 7\end{array}$ & $\begin{array}{l}18 \cdot 9 \\
21 \cdot 0 \\
23 \cdot 1\end{array}$ \\
\hline $1 \cdot 5800$ & $\begin{array}{l}\text { 1st } \\
\text { Mean } \\
\text { 99th }\end{array}$ & $\begin{array}{l}26 \cdot 2 \\
27.9 \\
29.6\end{array}$ & $\begin{array}{l}25 \cdot 7 \\
27 \cdot 3 \\
28 \cdot 9\end{array}$ & $\begin{array}{l}25 \cdot 1 \\
26 \cdot 6 \\
28 \cdot 2\end{array}$ & $\begin{array}{l}24 \cdot 5 \\
26 \cdot 0 \\
27 \cdot 5\end{array}$ & $\begin{array}{l}23 \cdot 9 \\
25 \cdot 3 \\
26 \cdot 8\end{array}$ & $\begin{array}{l}23 \cdot 2 \\
24 \cdot 7 \\
26 \cdot 1\end{array}$ & $\begin{array}{l}22 \cdot 6 \\
24 \cdot 0 \\
25 \cdot 5\end{array}$ & $\begin{array}{l}21 \cdot 9 \\
23 \cdot 4 \\
24 \cdot 9\end{array}$ & $\begin{array}{l}21 \cdot 1 \\
22 \cdot 7 \\
24 \cdot 3\end{array}$ & $\begin{array}{l}20 \cdot 4 \\
22 \cdot 1 \\
23 \cdot 7\end{array}$ \\
\hline $1 \cdot 6200$ & $\begin{array}{l}\text { 1st } \\
\text { Mean } \\
99 \text { th }\end{array}$ & $\begin{array}{l}27 \cdot 5 \\
28 \cdot 8 \\
30 \cdot 1\end{array}$ & $\begin{array}{l}27 \cdot 0 \\
28 \cdot 2 \\
29 \cdot 4\end{array}$ & $\begin{array}{l}26 \cdot 4 \\
27 \cdot 5 \\
28 \cdot 6\end{array}$ & $\begin{array}{l}25 \cdot 8 \\
26.9 \\
27.9\end{array}$ & $\begin{array}{l}25 \cdot 2 \\
26 \cdot 2 \\
27 \cdot 2\end{array}$ & $\begin{array}{l}24 \cdot 6 \\
25.6 \\
26.6\end{array}$ & $\begin{array}{l}23 \cdot 9 \\
24 \cdot 9 \\
26 \cdot 0\end{array}$ & $\begin{array}{l}23 \cdot 1 \\
24 \cdot 2 \\
25 \cdot 4\end{array}$ & $\begin{array}{l}22 \cdot 4 \\
23 \cdot 6 \\
24 \cdot 8\end{array}$ & $\begin{array}{l}21 \cdot 6 \\
22 \cdot 9 \\
24 \cdot 3\end{array}$ \\
\hline $1 \cdot 6600$ & $\begin{array}{l}\text { 1st } \\
\text { Mean } \\
\text { 99th }\end{array}$ & $\begin{array}{l}28 \cdot 6 \\
29.6 \\
30.6\end{array}$ & $\begin{array}{l}28 \cdot 0 \\
29 \cdot 0 \\
30.0\end{array}$ & $\begin{array}{l}27 \cdot 5 \\
28 \cdot 3 \\
29 \cdot 1\end{array}$ & $\begin{array}{l}26 \cdot 9 \\
27 \cdot 6 \\
28 \cdot 3\end{array}$ & $\begin{array}{l}26 \cdot 2 \\
27 \cdot 0 \\
27 \cdot 7\end{array}$ & $\begin{array}{l}25 \cdot 6 \\
26 \cdot 3 \\
27 \cdot 0\end{array}$ & $\begin{array}{l}24 \cdot 8 \\
25 \cdot 6 \\
26 \cdot 5\end{array}$ & $\begin{array}{l}24 \cdot 1 \\
25 \cdot 0 \\
25 \cdot 9\end{array}$ & $\begin{array}{l}23 \cdot 3 \\
24 \cdot 3 \\
25 \cdot 4\end{array}$ & $\begin{array}{l}22 \cdot 5 \\
23 \cdot 7 \\
24 \cdot 9\end{array}$ \\
\hline $1 \cdot 7000$ & $\begin{array}{l}\text { 1st } \\
\text { Mean } \\
\text { 99th }\end{array}$ & $\begin{array}{l}29 \cdot 3 \\
30 \cdot 2 \\
31 \cdot 0\end{array}$ & $\begin{array}{l}28 \cdot 8 \\
29 \cdot 5 \\
30 \cdot 2\end{array}$ & $\begin{array}{l}28 \cdot 2 \\
28 \cdot 8 \\
29 \cdot 5\end{array}$ & $\begin{array}{l}27 \cdot 6 \\
28 \cdot 2 \\
28 \cdot 8\end{array}$ & $\begin{array}{l}27 \cdot 0 \\
27 \cdot 5 \\
28 \cdot 1\end{array}$ & $\begin{array}{l}26 \cdot 2 \\
26 \cdot 9 \\
27 \cdot 5\end{array}$ & $\begin{array}{l}25 \cdot 5 \\
26 \cdot 2 \\
27 \cdot 0\end{array}$ & $\begin{array}{l}24 \cdot 7 \\
25.6 \\
26.5\end{array}$ & $\begin{array}{l}23.9 \\
24.9 \\
25.9\end{array}$ & $\begin{array}{l}23 \cdot 1 \\
24 \cdot 3 \\
25 \cdot 5\end{array}$ \\
\hline $1 \cdot 7400-$ & $\begin{array}{l}\text { 1st } \\
\text { Mean } \\
\text { 99th }\end{array}$ & $\begin{array}{l}29.8 \\
30.6 \\
31.4\end{array}$ & $\begin{array}{l}29 \cdot 3 \\
29.9 \\
30.6\end{array}$ & $\begin{array}{l}28 \cdot 7 \\
29 \cdot 3 \\
29 \cdot 8\end{array}$ & $\begin{array}{l}28 \cdot 1 \\
28 \cdot 6 \\
29 \cdot 1\end{array}$ & $\begin{array}{l}27 \cdot 4 \\
28 \cdot 0 \\
28 \cdot 5\end{array}$ & $\begin{array}{l}26 \cdot 7 \\
27 \cdot 3 \\
27 \cdot 9\end{array}$ & $\begin{array}{l}25 \cdot 9 \\
26 \cdot 7 \\
27 \cdot 4\end{array}$ & $\begin{array}{l}25 \cdot 1 \\
26 \cdot 0 \\
26 \cdot 9\end{array}$ & $\begin{array}{l}24 \cdot 3 \\
25 \cdot 3 \\
26 \cdot 4\end{array}$ & $\begin{array}{l}23 \cdot 5 \\
24 \cdot 7 \\
25 \cdot 9\end{array}$ \\
\hline $1 \cdot 7800$ & $\begin{array}{l}\text { 1st } \\
\text { Mean } \\
\text { 99th }\end{array}$ & $\begin{array}{l}30.2 \\
30.9 \\
31.6\end{array}$ & $\begin{array}{l}29 \cdot 6 \\
30 \cdot 2 \\
30 \cdot 8\end{array}$ & $\begin{array}{l}29 \cdot 0 \\
29 \cdot 5 \\
30 \cdot 1\end{array}$ & $\begin{array}{l}28 \cdot 4 \\
28.9 \\
29 \cdot 4\end{array}$ & $\begin{array}{l}27 \cdot 7 \\
28 \cdot 2 \\
28 \cdot 8\end{array}$ & $\begin{array}{l}26 \cdot 9 \\
27 \cdot 6 \\
28 \cdot 2\end{array}$ & $\begin{array}{l}26 \cdot 1 \\
26 \cdot 9 \\
27 \cdot 7\end{array}$ & $\begin{array}{l}25 \cdot 3 \\
26 \cdot 3 \\
27 \cdot 2\end{array}$ & $\begin{array}{l}24 \cdot 5 \\
25 \cdot 6 \\
26 \cdot 7\end{array}$ & $\begin{array}{l}23 \cdot 7 \\
25 \cdot 0 \\
26 \cdot 3\end{array}$ \\
\hline $1 \cdot 8200$ & $\begin{array}{l}\text { 1st } \\
\text { Mean } \\
\text { 99th }\end{array}$ & $\begin{array}{l}30 \cdot 3 \\
31 \cdot 0 \\
31 \cdot 7\end{array}$ & $\begin{array}{l}29 \cdot 7 \\
30.3 \\
30.9\end{array}$ & $\begin{array}{l}29 \cdot 2 \\
29 \cdot 7 \\
30 \cdot 2\end{array}$ & $\begin{array}{l}28 \cdot 5 \\
29 \cdot 0 \\
29 \cdot 5\end{array}$ & $\begin{array}{l}27 \cdot 8 \\
28.4 \\
29.0\end{array}$ & $\begin{array}{l}27 \cdot 0 \\
27 \cdot 7 \\
28 \cdot 4\end{array}$ & $\begin{array}{l}26 \cdot 2 \\
27 \cdot 1 \\
27 \cdot 9\end{array}$ & $\begin{array}{l}25 \cdot 4 \\
26 \cdot 4 \\
27 \cdot 4\end{array}$ & $\begin{array}{l}24 \cdot 6 \\
25 \cdot 7 \\
26 \cdot 9\end{array}$ & $\begin{array}{l}23 \cdot 7 \\
25 \cdot 1 \\
26 \cdot 4\end{array}$ \\
\hline $1 \cdot 8600$ & $\begin{array}{l}\text { Ist } \\
\text { Mean } \\
\text { 99th }\end{array}$ & $\begin{array}{l}30 \cdot 2 \\
30.9 \\
31 \cdot 7\end{array}$ & $\begin{array}{l}29 \cdot 6 \\
30 \cdot 3 \\
31 \cdot 0\end{array}$ & $\begin{array}{l}29.0 \\
29.6 \\
30.2\end{array}$ & $\begin{array}{l}28 \cdot 3 \\
29 \cdot 0 \\
29 \cdot 6\end{array}$ & $\begin{array}{l}27 \cdot 6 \\
28 \cdot 3 \\
29 \cdot 0\end{array}$ & $\begin{array}{l}26 \cdot 8 \\
27 \cdot 7 \\
28 \cdot 5\end{array}$ & $\begin{array}{l}26 \cdot 1 \\
27 \cdot 0 \\
28 \cdot 0\end{array}$ & $\begin{array}{l}25 \cdot 2 \\
26 \cdot 4 \\
27 \cdot 5\end{array}$ & $\begin{array}{l}24 \cdot 4 \\
25 \cdot 7 \\
27 \cdot 0\end{array}$ & $\begin{array}{l}23 \cdot 6 \\
25 \cdot 0 \\
26 \cdot 5\end{array}$ \\
\hline $1 \cdot 9000$ & $\begin{array}{l}\text { lst } \\
\text { Mean } \\
\text { 99th }\end{array}$ & $\begin{array}{l}29 \cdot 8 \\
30.7 \\
31.7\end{array}$ & $\begin{array}{l}29 \cdot 2 \\
30 \cdot 1 \\
31 \cdot 0\end{array}$ & $\begin{array}{l}28 \cdot 5 \\
29.4 \\
30 \cdot 3\end{array}$ & $\begin{array}{l}27 \cdot 9 \\
28 \cdot 8 \\
29 \cdot 7\end{array}$ & $\begin{array}{l}27 \cdot 2 \\
28 \cdot 1 \\
29 \cdot 1\end{array}$ & $\begin{array}{l}26 \cdot 4 \\
27 \cdot 5 \\
28 \cdot 5\end{array}$ & $\begin{array}{l}25 \cdot 6 \\
26 \cdot 8 \\
28 \cdot 0\end{array}$ & $\begin{array}{l}24 \cdot 9 \\
26 \cdot 2 \\
27 \cdot 5\end{array}$ & $\begin{array}{l}24 \cdot 1 \\
25 \cdot 5 \\
27 \cdot 0\end{array}$ & $\begin{array}{l}23 \cdot 3 \\
24 \cdot 9 \\
26 \cdot 5\end{array}$ \\
\hline $\begin{array}{r}1 \cdot 9400 \\
-1 \cdot 9799\end{array}$ & $\begin{array}{l}\text { 1st } \\
\text { Mean } \\
\text { 99th }\end{array}$ & $\begin{array}{l}29.0 \\
30.4 \\
31.8\end{array}$ & $\begin{array}{l}28 \cdot 4 \\
29 \cdot 7 \\
31 \cdot 1\end{array}$ & $\begin{array}{l}27 \cdot 8 \\
29 \cdot 1 \\
30 \cdot 4\end{array}$ & $\begin{array}{l}27 \cdot 1 \\
28 \cdot 4 \\
29 \cdot 8\end{array}$ & $\begin{array}{l}26 \cdot 5 \\
27 \cdot 8 \\
29 \cdot 1\end{array}$ & $\begin{array}{l}25 \cdot 7 \\
27 \cdot 1 \\
28 \cdot 6\end{array}$ & $\begin{array}{l}24 \cdot 9 \\
26 \cdot 5 \\
28 \cdot 0\end{array}$ & $\begin{array}{l}24 \cdot 2 \\
25 \cdot 8 \\
27 \cdot 4\end{array}$ & $\begin{array}{l}23 \cdot 4 \\
25 \cdot 2 \\
27 \cdot 0\end{array}$ & $\begin{array}{l}22 \cdot 6 \\
24 \cdot 5 \\
26 \cdot 4\end{array}$ \\
\hline
\end{tabular}

The product moment zero order correlation coefficients between each pair of variates where the relationship is linear are as shown in Table IX.

TABLE $\mathbf{X}$

CORRELATION COEFFICIENTS

\begin{tabular}{|c|c|c|c|c|c|}
\hline \multirow{2}{*}{\multicolumn{2}{|c|}{ Variates }} & \multicolumn{2}{|c|}{ Men } & \multicolumn{2}{|c|}{ Women } \\
\hline & & Right & Left & Right & Left \\
\hline Hand grip pressure and log weight . & .. & +0.4307 & +0.4541 & & \\
\hline Hand grip pressure and age & $\ldots$ & -0.4776 & -0.4429 & -0.4334 & -0.4663 \\
\hline Log weight and age ... & .. & \multicolumn{2}{|c|}{-0.0765} & \multicolumn{2}{|c|}{-0.3177} \\
\hline
\end{tabular}


These correlation coefficients, except log weight and age for men, are highly significantly different from zero $(P<0.01)$. The sex contrast between the significant correlation coefficient of -0.3177 for log weight and age in women and the non-significant value of -0.0765 in men may reflect the absence of obese men in this series.

The coefficients of multiple correlation are as follows:

\begin{tabular}{l|c|c}
\hline Hand & Men & Women \\
\hline Right & 0.6003 & 0.4615 \\
Left & 0.6132 & 0.5177 \\
\hline
\end{tabular}

These values though significantly different from zero are somewhat small. They indicate that, while body weight and age are of value in assessing hand grip pressure, it is desirable to seek additional effective correlatives.

\section{SUMMaRY}

(1) The hand grip pressure of 362 men and 394 women considered to be healthy and aged 60 to 89 years is assessed with reference to body weight and age.

(2) In both sexes hand grip pressure is significantly related to body weight and age. The important equations are:

$$
\begin{array}{ccc}
\text { MEN } & \text { Right } & Y=32.945028 X_{1}-0.32700776 X_{2} \\
& +3.953282 & \\
& \text { Left } & Y=33.905594 X_{1}-0.31281065 X_{2}
\end{array}
$$

$$
\begin{array}{ccc}
\text { WOMEN } & \text { Right } & Y=146.988935 X_{1}-39.728208 X_{1}^{2} \\
& -0.1990848 X_{2}-91.23304 \\
& \text { Left } & Y=179.298176 X_{1}-48.448874 X_{1}^{2} \\
& & -0.2181515 X_{2}-121.48802
\end{array}
$$

where $Y=$ hand grip pressure (kg.), $X_{1}=$ body weight (log kg.), and $X_{2}=$ age (completed years).

(3) The means, first and ninety-ninth percentiles of hand grip pressure in terms of body weight and age are presented.

We should like to acknowledge with grateful thanks advice on statistical methods given by $\mathrm{Dr} R$. A. Robb, Mitchell Lecturer on Methods of Statistics, Glasgow University.

Acknowledgement is made to the Secretary of State for Scotland for a grant provided on the recommendation of the Advisory Committee on Medical Research.

\section{REFERENCES}

Anderson, A. B. (1948). Cited in "The Practice of Endocrinology". p. 319. Eyre \& Spottiswoode, London.

Anderson, W. F., and Cowan, N. R. (1955). Lancet, 2, 239.

Burke, W. E., Tuttle, W. W., Thompson, C. W., Janney, C. D., and Weber, R. J. (1953). J. appl. Physiol., 5, 628.

Elderton, E. M., and Moul, M. (1928). Ann. Eugen. (Lond.), 3, 277.'

Fisher, M. B., and Birren, J. E. (1947). J. appl. Psychol., $31,490$.

Levine, S. A., and Harvey, W. P. (1949). "Clinical Auscultation of the Heart", p. 145. Saunders, Philadelphia.

Ruger, H. A., and Stoessiger, B. (1927). Ann. Eugen. (Lond.), $2,76$. 